\title{
En Face Optical Coherence Tomography of Inner Retinal Defects after Internal Limiting Membrane Peeling for Idiopathic Macular Hole
}

\author{
Micol Alkabes, ${ }^{1,2}$ Cecilia Salinas, ${ }^{1}$ Lucia Vitale, ${ }^{2}$ Anniken Burés-Jelstrup, ${ }^{1}$ Paolo Nucci, ${ }^{2}$ \\ and Carlos Mateo ${ }^{1}$
}

Purpose. To describe the appearance of inner retinal defects using en face spectral domain optical coherence tomography (SD-OCT) after idiopathic full-thickness macular hole (FTMH) surgery, referred to as concentric macular dark spots (CMDS).

Methods. In a retrospective cohort study, the authors evaluated 36 eyes of 36 patients with large idiopathic $\mathrm{MH}(>400$ $\mu \mathrm{m})$ who underwent standard three-port pars plana vitrectomy with internal limiting membrane (ILM) peeling. All patients were analyzed using B-scan and C-scan en face SD-OCT before and after surgery to determinate the OCT pattern of the retinal surface.

Results. Mean follow-up was 10 months ( \pm 8.45 SD; range, 3-30 months). Three months after surgery 36 of 36 eyes (100\%) showed a CMDS appearance on en face SD-OCT images. Anatomic success rate was 100\% (36/36 eyes) after a single surgical procedure. Once they were evident, these dark spots observed on the retinal surface were not progressive and remained stable over time.

Conclusions. Inner retinal defects frequently occurred after idiopathic MH surgery when ILM was peeled. To the authors' knowledge, this feature has not been previously reported using an en face SD-OCT imaging, and it consisted of numerous CMDS in the same direction of the optic nerve fibers. All patients in the study showed this typical OCT pattern 3 months after surgery. Thus, the authors suggest that this is a helpful, noninvasive technique to assess complete ILM removal in FTMH surgery if CMDS appearance on the retinal surface is reported. (Invest Ophthalmol Vis Sci. 2011;52:8349-8355) DOI:10.1167/iovs.11-8043

$P^{3}$ ars plana vitrectomy (PPV) and ILM peeling are standard procedure for idiopathic full-thickness macular hole (FTMH) surgery. Kelly and Wendel in $1991^{1}$ first reported successful closure after PPV with the creation of a posterior vitreous detachment placement of a gas bubble with at least 1 or 2 weeks of face-down positioning. In 1996 Liesenhoff et al. ${ }^{2}$ showed that the removal of any epiretinal membrane associated with an idiopathic FTMH significantly improved the surgical outcome. Later, some authors ${ }^{3,4}$ demonstrated higher hole closure rates and functional gain with ILM peeling in MH surgery because of the hypothesis that the ILM also exerted

From the ${ }^{1}$ Instituto de Microcirugía Ocular, Barcelona, Spain; and the ${ }^{2}$ San Giuseppe Hospital Eye Clinic, Milan, Italy.

Submitted for publication June 13, 2011; revised August 8, 2011; accepted August 8, 2011

Disclosure: M. Alkabes, None; C. Salinas, None; L. Vitale, None; A. Burés-Jelstrup, None; P. Nucci, None; C. Mateo, None

Corresponding author: Micol Alkabes, San Giuseppe Hospital, Eye Clinic, Via San Vittore, 12, 20123 Milan, Italy;

micol_alkabes@hotmail.com. some degree of tangential traction on the edges of the hole. Relief of this traction was necessary to close it successfully.

Anatomic changes of the macula after PPV with removal of ILM are described in the literature, though they were considered infrequent. ${ }^{5}$ They consisted primarily of paracentral scotomas observed by SLO microperimetry, retinal pigment epithelium (RPE) changes, and dissociated optic nerve fibers layer (DONFL), as reported on fundus photographs. ${ }^{6,7}$

The DONFL appearance we refer to in this study was first described by Tadayoni et al. in $2001 .^{8}$ They reported the unique appearance of the fundus after epiretinal membrane (ERM) removal, presumably related to ILM peeling. Using bluefilter photographs, they described the frequent feature of arcuate, slightly dark striae within the posterior pole along the course of optic nerve fibers, and they called this the DONFL appearance. The same feature was also faintly visible on redfree photographs and on fundus examination.

It was reported later that B-scans of optical coherence tomography (OCT) revealed dimples in the retinal nerve fiber layer (RNFL) corresponding to each stria of the DONFL appearance and that the depths of all the dimples were limited to the RNFL thickness. ${ }^{9,10}$

Indeed, OCT is a well-established tool for diagnosis and follow-up of vitreoretinal disorders. Conventional time-domain OCT, however, with its approximately $10-\mu \mathrm{m}$ axial resolution, has limited ability to show details. The introduction of highresolution spectral-domain (SD) OCT, with an approximately $5-\mu \mathrm{m}$ axial resolution, highly improved the visualization of details in intraretinal layers and vitreoretinal interface. Moreover, some SD-OCT can provide particular scan modes, such as en face C-scans, which offer a new view of the different layers of the retina and the vitreoretinal relationship.

In this study, we retrospectively reviewed cases of FTMH surgery with ILM peeling using en face SD-OCT images to report the DONFL appearance, as described by Tadayoni. ${ }^{8}$ We identified a pattern of multiple dark dots along the course of the optic nerve fiber layer, and we called this feature concentric macular dark spots (CMDS). To our knowledge, this appearance using en face SD-OCT imaging has not been reported in the literature until now.

\section{Methods}

In a retrospective cohort study, we evaluated 36 eyes of 36 patients who underwent standard three-port pars plana vitrectomy for idiopathic FTMH (larger than $400 \mu \mathrm{m}$ ) between 2007 and 2010. All examinations and investigations adhered to the tenets of the Declaration of Helsinki.

Patients with myopic and traumatic MH were excluded. All patients were evaluated at the Instituto de Microcirugia Ocular (Barcelona, Spain), and surgery was carried out by the same surgeon (CM). 
In all cases, B-scans and C-scan en face spectral domain OCT (Cirrus HD-OCT 4000, version 5.0; Carl Zeiss Meditec, Dublin, CA) were performed before and after surgery to establish the characteristic OCT pattern of the DONFL appearance. All the study eyes were also evaluated using a mydriatic fundus camera (TRC-50DX type IA; Topcon, Tokyo, Japan) in association with OCT images.

All patients underwent SD-OCT 3 months after surgery, though most of them were also evaluated with OCT 1 month after surgery and long afterward during their routine ophthalmic examinations.

B- and C-scans were acquired, before and after surgery, using the HD 5 Line Raster for horizontal and vertical images and Macular Cube $512 \times 128$ to create the en face image. MH size was considered the maximum diameter of the hole and was measured at the level of the RPE. Advanced Visualization, an interactive multiplanar reformat that enables viewing of image cross-sections through three dimensions, was selected in the Analysis List on Cirrus HD-OCT 4000.

In the screen view obtained, the upper left viewport showed the saved fundus image, whereas the other three viewports showed cross sectional scan images in three planes. If they are thought of as a cube, the images are shown in the viewports as planes parallel to the side (Y plane, lower left viewport), to the front (X plane, upper right viewport), and to the top ( $\mathrm{Z}$ plane, lower right viewport) of the cube. Then, on the Overlay drop-down options, None was selected to show the corresponding saved fundus image with any slices (upper left image)

Knowing that cube scan analyses incorporate an algorithm to automatically find and display the inner limiting membrane (ILM), the RPE, and RPEfit, in the lower right viewport and on the drop-down options, it was necessary to change the default setting (Slice) by selecting the $I L M$ layer, which corresponds to the en face image on the $\mathrm{Z}$ plane of the macular cube. Finally, to improve image quality, grayscale of slices was preferred.

Surgery consisted of a standard 23-gauge, three-port PPV with induced posterior vitreous detachment, when absent, and ILM peeling in all cases. Detachment and removal of the posterior hyaloid was performed to the mid-periphery (trying to avoid peripheral breaks), and the ILM was peeled with the end-gripping forceps to the vascular arcades. The surgeon judged whether the ILM was complete, as defined by removal of the ILM $360^{\circ}$ all around the hole within a minimal extension of approximately 2 or 3 disc diameters centered on the center of the hole. Dye-assisted peeling of ERM, if present, and of ILM was performed using $0.2 \mathrm{~mL}$ highly purified trypan blue (MembranBlue; DORC, Zuidland, The Netherlands), brilliant blue G (BrilliantPeel; Geuder, Heidelberg, Germany), or both. After a first fluid-air exchange, with some fluid left on the retinal surface at the posterior pole to avoid dye dispersion into the vitreous cavity, dyes were applied in an airfilled eye for approximately 2 minutes. Agents were washed out by a second air-fluid exchange, and ERM/ILM was subsequently peeled. Restaining was permitted but was not necessary in any case. Finally, a third fluid-air exchange with injection of $20 \%$ sulfur hexafluoride $\left(\mathrm{SF}_{6}\right)$ tamponade was performed. Patients were asked to maintain a prone position for 5 days after surgery.

MH was considered to be closed 3 months after surgery when it was no longer visible clinically and on OCT (open holes with or without subretinal fluid around them were considered to be open).

\section{Results}

The study included 36 eyes of 36 patients with FTMH who underwent PPV with a 23-gauge system and with dye-assisted ERM/ILM peeling between 2007 and 2010. The mean follow-up was 10 months ( $\pm 8.45 \mathrm{SD}$; range, $3-30$ months). Based on the Gass classification, ${ }^{11} \mathrm{MH}$ stage $>400 \mu \mathrm{m}$ was stage $3(n=16)$ and stage $4(n=20)$. Mean hole diameter was $782.58 \mu \mathrm{m} \pm$ 302.64 SD (range, 419-1672 $\mu \mathrm{m}$ ).

The DONFL appearance, which Tadayoni et al. ${ }^{8}$ first described as arcuate and slightly dark striae within the posterior pole along the course of optic nerve fibers, was found on en face SD-OCT (Cirrus HD-OCT) in a total of 36 of 36 eyes (100\%) 3 months after surgery. We called this feature CMDS because of the appearance, on the en face SD-OCT images, of multiple dark dots along the course of retinal nerve fibers in the area of the ILM peeling (Fig. 1).

No CMDS appearance was evident before surgery (Figs. 2-4). Most of the patients also underwent tomographic examination with en face C-scans long afterward, but none showed any substantial change in CMDS appearance (Fig. 3). Moreover, the anatomic success rate was $100 \%$ (36/36 eyes)
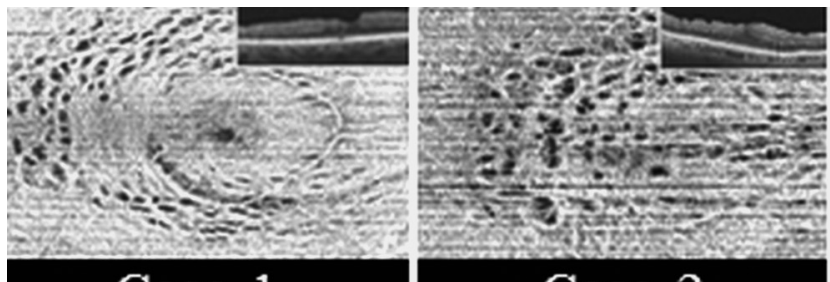

\section{Case 1}

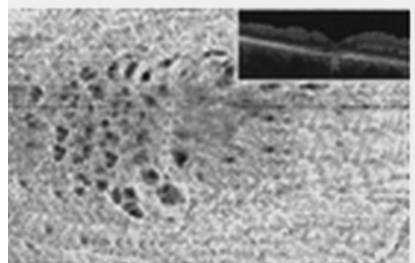

\section{Case 4}

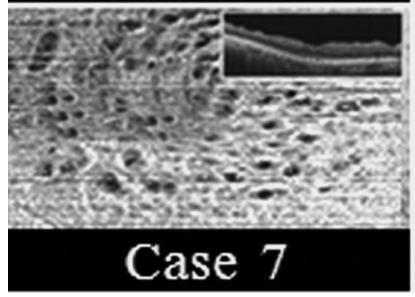

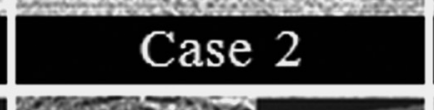
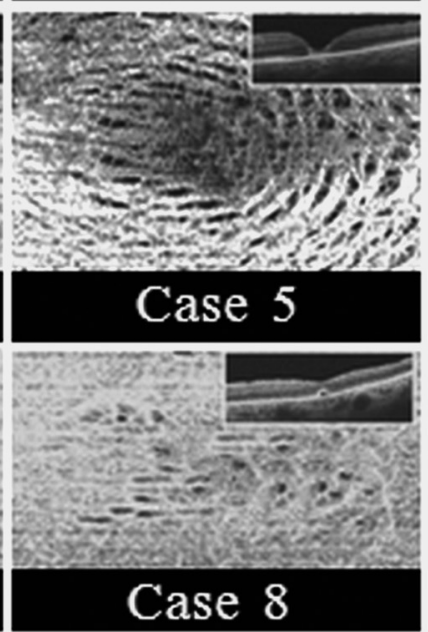

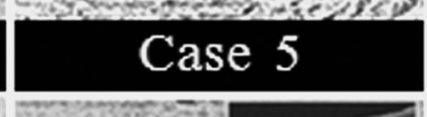

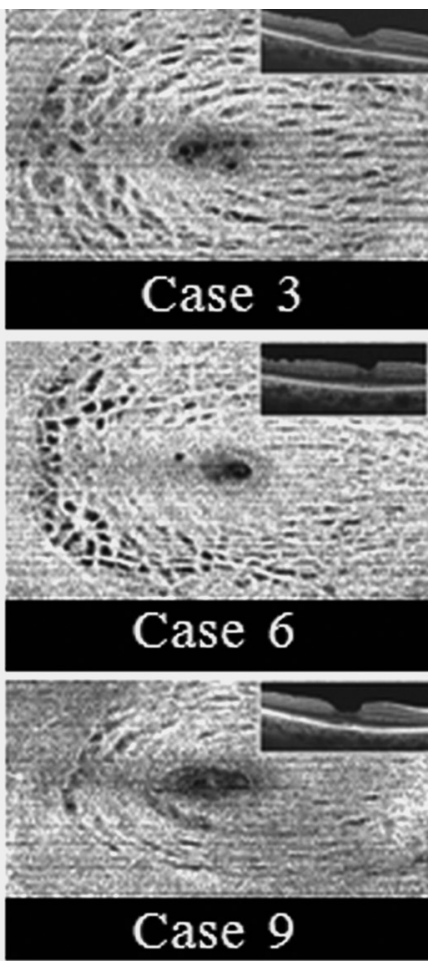

FIGURE 1. Three-month postoperative en face SD-OCT with horizontal B-scans of nine patients who underwent PPV with ILM peeling for FTMH. Multiple dark dots along the course of optic nerve fiber layer were clearly visible in the area of the peeled ILM. 
FIGURE 2. Preoperative en face SDOCT (A) and horizontal B-scan (B) of an FTMH with a detached posterior hyaloid. En face SD-OCT C-scans (C) were also acquired 3 months after surgery showing a characteristic OCT pattern with the CMDS appearance, which was not observed before surgery. Complete MH closure (D) with an interrupted IS/OS line and an intact ELM 3 months after surgery (asterisks). Some focal dehiscences, corresponding to each dark spot, were visible in the RNFL on B-scan OCT images (white arrows)
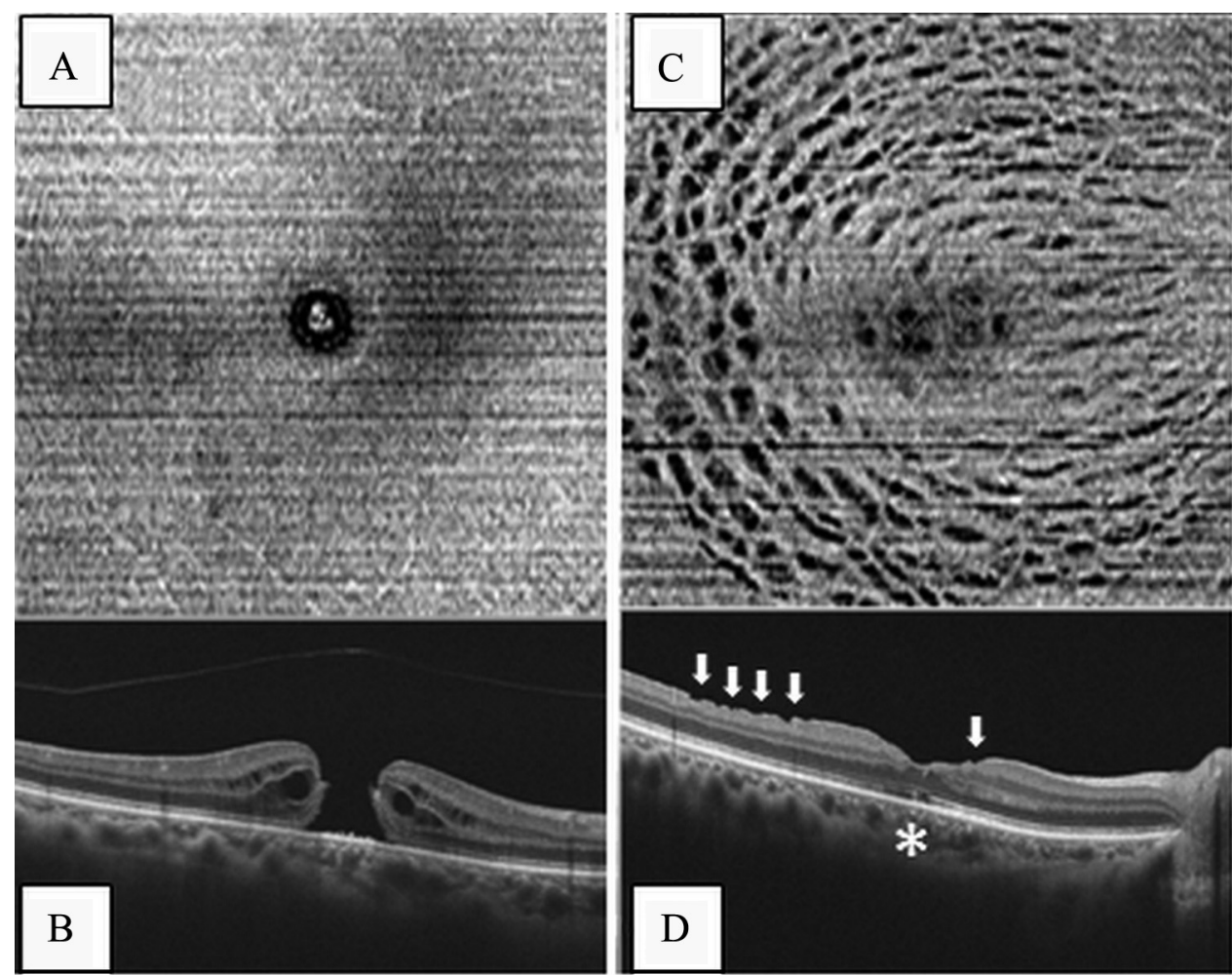

after a single surgical procedure and in some cases, complete closure of the MH was observed on OCT B-scans within 1 day.

\section{Discussion}

Spectral domain acquisition allows the Cirrus HD-OCT 4000 to acquire images at 27,000 axially oriented A-scans per second compared with its time domain predecessor, which acquired $400 \mathrm{~A}-\mathrm{scan} / \mathrm{s}$. This high-resolution SD-OCT, which has been used in ophthalmic clinical practice since 2004, makes use of a low-coherence superluminescent diode light source at a near infrared wavelength of $840 \mathrm{~nm}$ with a bandwidth of $50 \mathrm{~nm}$, achieving $5-\mu \mathrm{m}$ axial resolution and $15-\mu \mathrm{m}$ transverse resolution in tissue. ${ }^{12}$

Two standard scan protocols were used to acquire data: a $512 \times 128$ cube and a frame-averaged cross-sectional scan through the fovea. The first one consisted of 128 cross-sectional images, or B-scans, each composed of 512 A-scans. The second one was obtained using the system's commercial software (version 5.0), which averages 20 individual B-scans, each consisting of 1024 A-scans in which the nominal spacing between scans was set to zero.

In the en face OCT, the transversal scanner produces the fast lines in the image. ${ }^{13}$ Whereas B-scans produce cross-sectional images of retinal morphology, bearing a strong resemblance to histology, OCT C-scans are represented as two-dimensional transversal slices at any given depth through the retina, thereby enabling visualization of the lateral extent of structures. ${ }^{14}$

These scans allow an en face transverse perspective, which is the view obtained by the sum of the C-scan planes indicated in the thickness value field, to produce a high-contrast image of the retina surface detail.

The ILM is one of the inner retinal structures that can be better analyzed by the en face OCT technique. Given that it might be involved in the pathogenesis of disorders affecting the vitreomacular surface, including the $\mathrm{MH}$, we decided to conduct a retrospective study to report some tomographic findings, such as the DONFL appearance, on the retinal surface after MH surgery with ILM removal.

The DONFL appearance after ILM peeling was described for the first time by Tadayoni et al. ${ }^{8}$ Using blue-filter photographs, they showed that it consisted of numerous slightly dark arcuate striae within the posterior pole, in the same direction of the optic nerve fibers, and they hypothesized that it might be caused by traction exerted on the retina during the removal of ERM and ILM.

In that study, this feature was found, postoperatively, in $43 \%$ of patients who underwent surgery for idiopathic ERM with ILM peeling. ${ }^{8}$ Miura et al., ${ }^{15}$ in a case report, described the same aspect after idiopathic FTMH surgery.

To our knowledge, this appearance using en face SD-OCT images has not been reported in literature until now. We called this feature CMDS because of the appearance, on the en face SD-OCT images, of multiple dark dots, presumably along the course of the optic nerve fiber layer in the area of the ILM peeling. We considered that this acronym could be practical and useful to refer to the inner retinal defects, as shown on OCT C-scans, regarding their distribution, localization, and reflectivity but not concerning the underlying nature of these changes on the retinal surface, which will be fully discussed successively in this section.

In our study, CMDS appearance on en face SD-OCT was found in 36 of 36 eyes (100\%) 3 months after surgery. Tomographic examinations with en face C-scan were not routinely performed before 1 month because of the presence of gas, which did not allow us to evaluate the retinal surface from the top, as is required with en face images. Therefore, it was impossible to establish whether this feature was already present immediately after surgery.

Most patients were evaluated with SD-OCT en face C-scans long after surgery, during their routine ophthalmic examination. No one had any substantial changes in CMDS appearance. Thus, we can reasonably affirm that, once this en face 


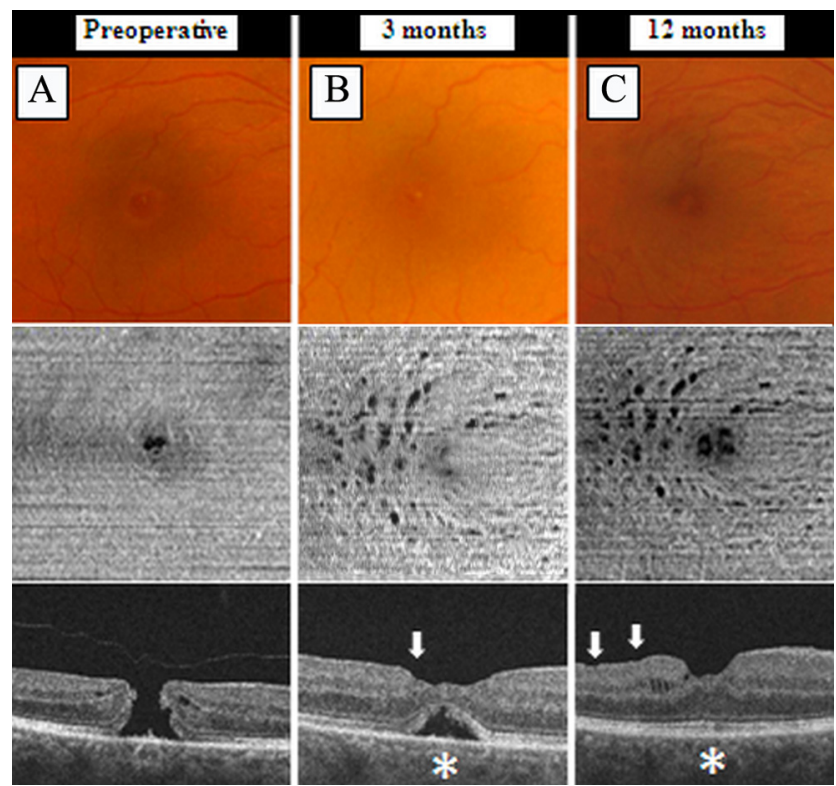

Figure 3. Twelve-month follow-up of a patient who underwent PPV with ILM removal for an idiopathic FTMH in the right eye. Color fundus photographs (top), en face SD-OCT C-scans (middle), and horizontal B-scans (bottom) were performed before surgery (A), 3 months after surgery (B), and 12 months after surgery (C). No CMDS was evident before surgery on en face images (A, middle left). At 3 months they were visible along the course of the optic nerve fibers layer, except at the level of the fovea where some degree of subretinal fluid was still present (B, middle). Twelve months after surgery, when subfoveal fluid has disappeared and foveal detachment has completely resolved, these inner retinal defects became even more well defined (C, middle right). Bottom, left to right: images confirm a preoperative FTMH with posterior vitreous detachment, its partial closure 3 months after surgery with a persistent foveal detachment, and its finally complete closure with return to the normal foveal architecture 12 months after surgery (asterisks). Dimples in the RNFL were observed after ILM removal (white arrows).

SD-OCT image is evident at 3 months, it becomes more clearly visible, it is not progressive, and it remains unchanged thereafter (Fig. 3).

In addition, regarding the postoperative tomographic features and concerning the recovery process after MH surgery, some of our patients presented outer foveal defects for a few months after surgery. Postoperative B-scans showed in the outer retina some hyporeflective cystic spaces, corresponding to a disrupted inner segment/outer segment (IS/OS) junction with a normal external limiting membrane (ELM). This feature suggests that the ELM may be the first structure to recover after MH closure. ${ }^{16}$ (Figs. 2, 4).

In accordance with Ito et al., ${ }^{10}$ although these postoperative changes on the inner retinal surface were difficult to appreciate during routine biomicroscopic fundus examination, they could be observed on color fundus photographs, as seen in Figure 4. Comparison of OCT images and blue-filter photographs also showed that the arcuate striae seen in blue-filter photographs seemed to correspond exactly with the focal dehiscences in the RNFL detected in OCT images. Thus, we suggest that the CMDS found on the en face SD-OCT view corresponded to the focal dehiscence in the thickness of the optic nerve fiber layer seen in B-scan OCT images (Figs. 2-4).

Mitamura et al. ${ }^{9}$ reported that the DONFL appearance was due to shallow dimples in the optic nerve fiber layer bundle, which are less deep than the thickness of RNFL.

Regarding the possible nature of these postoperative inner retinal defects, which we refer to as CMDS on OCT images,
Wolf et al. ${ }^{17}$ showed that indocyanine (ICG)-assisted ILM peeling itself caused minor, but histologically demonstrable, damage to the retinal surface. Using electron microscopy to analyze the peeled retina in two donor eyes, they described a substantial number of destroyed Müller cell endfeet and damaged cellular vitreal processes, even if no damage in the Müller cell perikaryon was found. Some degrees of retinal injury were also found even in the untouched retina directly adjacent to the peeled area as a result of stretching forces during the process of peeling. Moreover, evaluating the transition zone between the peeled and unpeeled area, they individuated some "empty spaces" that contained cellular debris, probably originating from Müller cell endfeet.

Since its introduction in clinical practice, several studies have been conducted to assess the in vivo toxicity of ICG on the retinal layers, ${ }^{18,19}$ and concerns have been raised over the possibility of RPE and retinal toxicity and the induction of apoptosis as a result of the exposure to ICG, especially with higher concentrations and contact times. ${ }^{20-22}$ In their experimental study on donor eyes, Wolf et al. ${ }^{17}$ did not discuss the relationship between this inner retinal damage and the use of ICG. Nevertheless, recent publications have confirmed the safety of ICG for MH surgery, including several large nonrandomized studies ${ }^{23-26}$ and one randomized clinical trial. ${ }^{6}$

The incidence of the DONFL appearance in cases of ILM staining by ICG did not seem to significantly differ from that without staining. ${ }^{10,27}$ This might suggest that ICG did not affect the incidence of the DONFL appearance, or of CMDS, although a histologic difference between an ICG-stained and an unstained ILM was found during its peeling.

As reported by Christensen et al., ${ }^{6}$ the DONFL appearance has the same incidence in ICG-peeled and Trypan blue-peeled eyes, suggesting that the ILM peeling procedure itself was responsible for this feature rather than the dye used.

Thus, assuming that they were considered at least minimally toxic to retinal cells and adhering to our clinical experience of a more challenging ILM peeling, blue dyes were preferred during our surgical technique, even though recent experimental studies have also reported possible TB toxicity in RPE cells. ${ }^{28-30}$

Moreover, Haritoglou et al. ${ }^{7}$ did not observe the DONFL appearance in approximately 100 patients who underwent ILM peeling, possibly because of the size of the ILM peeled area. In that study, the peeled area was approximately 1 disc diameter around the $\mathrm{MH}$, whereas we intended to perform the ILM peeling up to the vascular arcade (approximately 2 or 3 disc diameters). This may be why we found this feature in $100 \%$ of our peeled eyes, suggesting that the larger the area of ILM that is peeled, the more likely that CMDS (corresponding to the DONFL appearance) will be observed.

Because CMDS was not found in the preoperative OCT C-scans of patients with stage $4 \mathrm{MH}$, we could reasonably suppose that it is caused by ILM removal rather than by posterior hyaloid detachment, a procedure that does not involve touch. In addition, because all the patients showed CMDS on the postoperative en face SD-OCT images, it would be reasonable to assume that complete ILM peeling was performed within the posterior pole despite the impossibility of quantifying the area of peeled ILM. Given that this feature was not detected in $57 \%$ of ILM-peeled eyes, Tadayoni et al. ${ }^{8}$ suggested it might be due to incomplete ILM removal within the posterior pole. In this region, the attachment plaques between Müller cells are rarefied and the ILM is relatively thicker, whereas near the center of the fovea and toward to periphery it becomes thin, and the number of attachment plaques increases. ${ }^{31,32}$ Furthermore, this appearance seemed to be limited to the area of ILM peeling and was not detected in the surrounding normal 
Figure 4. Preoperative en face SDOCT (A) with no evidence of CMDS. Horizontal B-scan (B) of an FTMH with a retinal operculum on the posterior hyaloid. After surgery, CMDS appearance was observed on the en face images (C), and it seemed to correspond to each stria on color fundus photographs (D, lower right, white square). Anatomic success closure was confirmed by OCT B-scan, which also showed typical dehiscence at the level of the optic nerve fibers layer less deep than its total thickness (white arrows) and some foveal defects in the IS/OS line that could have occurred as a result of the healing process of MH surgery.
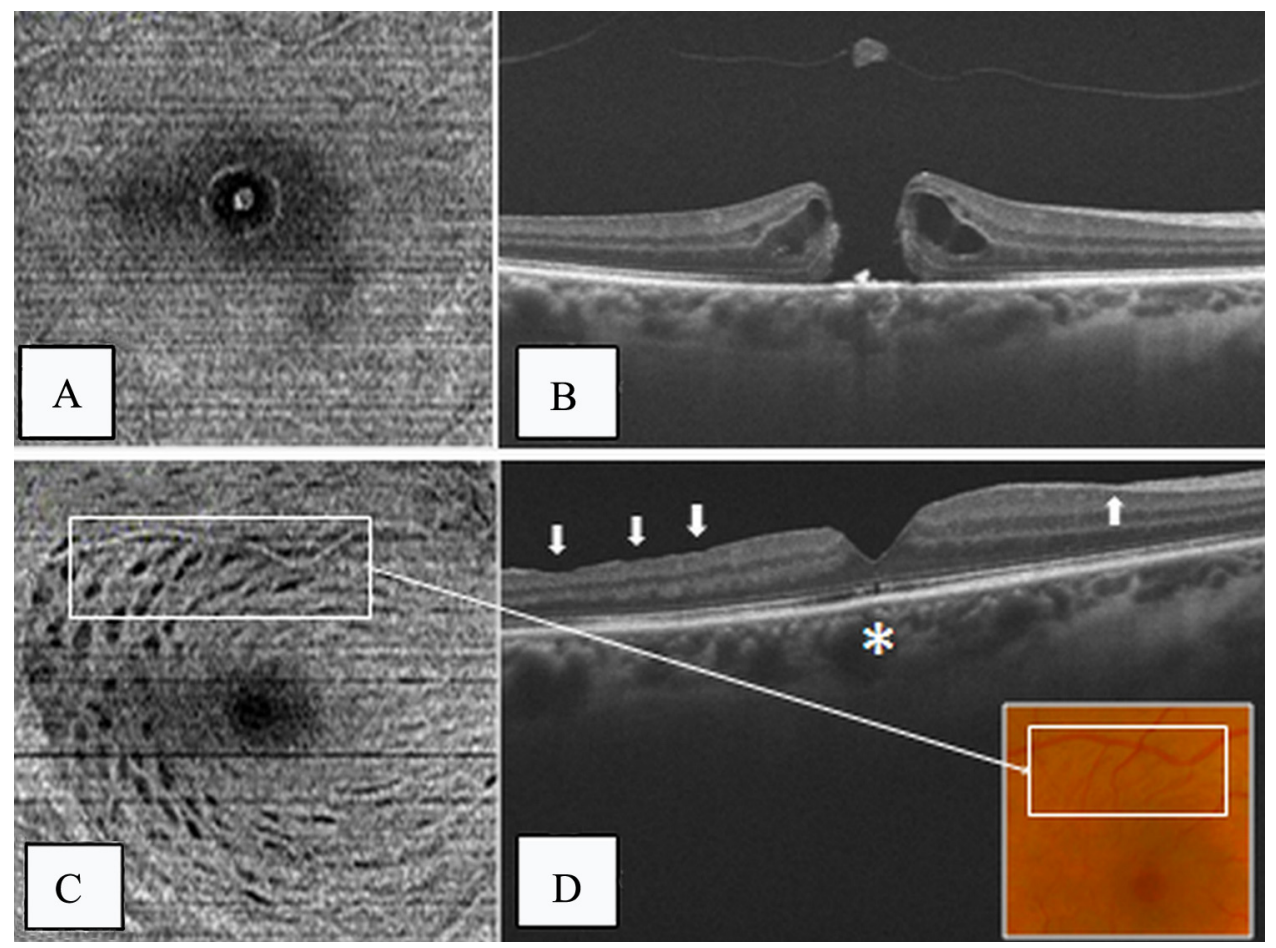

retina; further randomized prospective studies and histologic analyses are warranted.

The effects of ILM removal have been reported. Mitamura and Ohtsuka ${ }^{27}$ evaluated 74 eyes that underwent surgery for FTMH with ILM peeling. In their study, 46 of 74 eyes (62.2\%) exhibited the DONFL appearance on blue-filtered fundus photographs, but they did not find any significant difference between the zone of DONFL and the surrounding normal retina concerning the microperimetry threshold values, suggesting that there was no loss of optic nerve fibers. ${ }^{33}$ Ito et al. ${ }^{10}$ showed no significant difference in macular sensitivity measured by Humphrey 10-2 between ILM-peeled eyes with and without the DONFL appearance. Moreover, no difference was found between eyes with and without this feature with regard to preoperative and postoperative best-corrected visual acuity. ${ }^{10,27}$

Although Kim et al. ${ }^{34}$ reported some visual field defects in patients with ILM peeling, the DONFL appearance might not be associated with the loss of visual field in spite of what occurs in eyes with glaucoma, which show functional defects caused by nerve fiber loss.

However, Terasaki et al. ${ }^{35}$ suggested that ILM peeling during MH surgery could cause damage to Müller cells, which are in close contact with the ILM and maintain the nerve fiber bundles close to each other, as demonstrated by a limited and delayed response of the focal macular electroretinography (ERG). Later, Tari et al. ${ }^{36}$ demonstrated some degree of postoperative decrease in multifocal ERG response amplitudes. They did not attribute this effect to damage to the retinal nerve fiber layer or the ganglion cell layer, though those layers are closest to the ILM and may be affected by ILM peeling more than another retinal structures. After analyzing some tissue specimens, which included ILM, collagen fibers, and Müller cell footplate processes, Tari et al. ${ }^{36}$ suggested that dysfunction of Müller cells might play an important role in modulating synaptic transmission in the neural retinal circuitry.

In spite of these hypotheses, removal of ILM has been performed for years during FTMH surgery because that seems to increase the probability of successful closure of the hole. ILM peeling could prevent reopening of MH maybe due to the role of the ILM as a possible substratum on which glial tissue proliferates, causing further epiretinal membrane formation with progressive enlargement or reopening of the $\mathrm{MH}$ as a result of this contractile force.

Our study was not designed to assess the value of ILM peeling in MH surgery for its potential visual success, which can be shown by randomized clinical studies. Very interesting results have been obtained by The Full Thickness Macular Hole and Internal Limiting Membrane Peeling Study (FILMS), a multicentric randomized clinical trial in patients with stage 2 or 3 MH that compares two groups of patients (group with ILM peeling and group without ILM peeling). This study confirmed that a not statistically, but clinically significant, difference was found in favor of ILM peeling and a higher anatomic closure rate at 1 month (84\% in the ILM-peel group vs. $48 \%$ in the non-peel group) and a lower repeat operation rate within 6 months $(12 \%$ in the ILM-peel group vs. $48 \%$ in the non-peel group). No statistically significant difference in rates of hole closure between these two groups was observed at 3 and 6 months. Thus, these results could reasonably lead to the conclusion that ILM peeling is a highly cost-effective procedure primarily because of the low number of repeat operations. ${ }^{38}$

Anatomic results in FILMS are also comparable to those obtained in one of the recently publicized randomized clinical trials in which $\mathrm{MH}$ closure at 3 months was achieved in $44 \%$ and $89 \%$ in the non-peeled group and in the trypan blueassisted ILM group, respectively. ${ }^{6}$

These data are in contrast with the previous research conducted by Margherio et al., ${ }^{39}$ who showed no beneficial or adverse effects with ILM peeling in terms of hole closure rate or visual improvement. Therefore, they concluded that ILM removal was not essential.

Nevertheless, the benefits al ILM peeling have also been reported by another recently complete multicentric randomized clinical trial, which included only stage 3 or $4 \mathrm{MH}$ (larger than $400 \mu \mathrm{m}$ ) and which has been published until now only as 
an abstract (Tadayoni R, et al. IOVS. 2009;50:ARVO E-Abstract 5206).

Thus, based on these data, we reported a $100 \%$ anatomic success rate, indicating that ILM removal may be one of the most recent changes in the conventional surgical procedure to improve the rate of $\mathrm{MH}$ closure. Actually, this benefit may concern especially large holes $(>400 \mu \mathrm{m})$ for which the closure rate rose significantly when ILM was peeled off $(100 \%$ peeled vs. $56 \%-73 \%$ unpeeled). ${ }^{40,41}$

Moreover, no late reopening of a previously closed MH was observed during 3 years of follow-up, suggesting that ILM peeling could also be considered a safe and more cost-effective approach than no ILM peeling to treat stage 3 or 4 idiopathic FTMH, again because of the lower number of repeat operations.

In summary, we retrospectively reviewed 36 cases of FTMH surgery with ILM peeling using en face SD-OCT images to report the appearance of DONFL, as described first by Tadayoni et al. In our study, this feature was evident on OCT scans in $100 \%$ of patients 3 months after surgery. As far as we know, this feature has not been previously reported using an en face imaging of SD-OCT.

We are conscious that our study has several limitations, primarily because of its retrospective nature, which did not allow randomization into groups with and without ILM peeling ("no-touch" technique) to demonstrate which portion(s) of the MH procedure could be responsible for the appearance of inner retinal defects. However, this option seemed at least ethically inappropriate to us because of a significantly increased risk of lack of primary hole closure in the non-peeled patients with stage 3 or $4 \mathrm{MH}$.

Moreover, comparison of the OCT images of two different retinal areas of the same patient (one peeled and one unpeeled) would have allowed us to have an "internal control" group to assess these inner retinal defects related to ILM peeling, thereby adding a more solid base to our findings.

We did not evaluate any functional parameter, such as best-corrected visual acuity, visual field, or retinal sensibility, and this might be considered another limitation of our study; however, our purpose was to describe the presence of a characteristic OCT pattern that may often occur on the retinal surface after ILM peeling. Additional research and statistical analysis are needed to determinate whether a relationship might exist between these functional parameters and CMDS appearance.

Another weakness of our study might be the small number of patients. However, the same surgical technique was performed by the same surgeon, which reduces significantly any performance bias caused by different procedures.

In conclusion, we report this characteristic appearance on en face SD-OCT images in $100 \%$ of patients after idiopathic $\mathrm{MH}$ surgery at 3 months, and we have called this feature CMDS, the shape and the distribution of which suggest that they might be related to the course of the optic nerve fibers.

Thus, our study suggests that en face SD-OCT is a helpful, noninvasive technique to assess ILM peeling when CMDS appearance on the retinal surface is reported. We think this could be very important in cases of failed MH surgery, especially if no dye was used and if the ILM could still be adherent and maintaining tangential traction in those patients. ${ }^{42,43}$

Moreover, en face C-scans should be considered more accurate than blue-filter fundus photographs in detecting these retinal surface changes in ILM-peeled eyes (100\% vs. $54 \%-$ $62.2 \%$, respectively). ${ }^{9,10}$

\section{References}

1. Kelly NE, Wendel RT. Vitreous surgery for idiopathic macular holes: results of a pilot study. Arch Ophthalmol. 1991;109:654659.

2. Liesenhoff O, Messmer EM, Pulur A, Kampik A. Treatment of full thickness idiopathic macular holes. Opbthalmologe. 1996;93:655659.

3. Eckardt C, Eckardt U, Groos S, Luciano L, Reale E. Removal of the internal limiting membrane in macular holes: clinical and morphological findings. Opbthalmologe. 1997 Aug;94(8):545-551.

4. Brooks HL Jr. Macular hole surgery with and without internal limiting membrane peeling. Opbthalmology. 2000;107:19391948.

5. Haritoglou C, Gass C, Schaumberger M, Ehrt O, Gandorfer A, Kampik A. Macular changes after peeling of the internal limiting membrane in macular hole surgery. Am J Ophthalmol. 2001;132: 363-368.

6. Christensen UC, Kroyer K, Sander B, et al. Value of internal limiting membrane peeling in surgery idiopathic macular hole stage 2 and 3: a randomized clinical trial. Br J Opbthalmol. 2009;93:10051015.

7. Haritoglou C, Gandorfer A, Kampik A. NFL appearance after peeling. Opthalmology. 2006;113:1690.

8. Tadayoni R, Paques M, Massin P, et al. Dissociated optic nerve fiber layer appearance of the fundus after idiopathic epiretinal membrane removal. Ophthalmology. 2001;108:2279-2283.

9. Mitamura Y, Suzuki T, Kinoshita T, Miyano N, Tashimo A, Ohtsuka $\mathrm{K}$. Optical coherence tomographic findings of dissociated optic nerve fiber layer appearance. Am J Opbthalmol. 2004;137:11551156.

10. Ito Y, Terasaki H, Takahashi A, Yamakoshi T, Kondo M, Nakamura M. Dissociated optic nerve fiber layer after internal limiting membrane peeling for idiopathic macular holes. Ophthalmology. 2005; 112:1415-1420.

11. Gass JD. Idiopathic senile macular hole: its early stages and pathogenesis. Arch Opbthalmol. 1988;106:629-639.

12. Lujan BJ, Roorda A, Knighton RW, Carroll J. Revealing Henle's fiber layer using spectral domain optical coherence tomography. Invest Ophthalmol Vis Sci. 2011;52:1486-1492.

13. Bradu A, Podoleanu A, Rosen RB. High-speed en face optical coherence tomography system for the retina. $J$ Optoelectronics Adv Mater. 2005;7:2913-2918.

14. Forte R, Cennamo G, Pascotto F, de Crecchio G. En face optical coherence tomography of the posterior pole in high myopia. Am J Opbthalmol. 2008;145:281-288.

15. Miura M, Elsner AE, Osako M, Iwasaki T, Okano T, Usui M. Dissociated optic nerve fiber layer appearance after internal limiting membrane peeling for idiopathic macular hole. Retina. 2003;23: 561-563.

16. Bottoni F, De Angelis S, Luccarelli S, Cigada M, Staurenghi G. The dynamic healing process of idiopathic macular holes after surgical repair: a spectral-domain optical coherence tomography study. Invest Ophthalmol Vis Sci. 2011;52:4439-4446.

17. Wolf S, Schnurbusch U, Wiedemann P, Grosche J, Reichenbach A, Wolburg H. Peeling of the basal membrane in the human retina: ultrastructural effects. Opbthalmology. 2004;111:238-243.

18. Gandorfer A, Haritoglou C, Gass CA, Ulbig MW, Kampik A. Indocyanine green-assisted peeling of the internal limiting membrane may cause retinal damage. Am J Ophthalmol. 2001;132:431-433.

19. Gandorfer A, Haritoglou C, Gandorfer A, et al. Retinal damage from indocyanine green in experimental macular surgery. Invest Ophthalmol Vis Sci. 2003;44:316-323.

20. Engelbrecht NE, Freeman J, Sternberg, et al. Retinal pigment epithelial changes after macular hole surgery with indocyanine greenassisted internal limiting membrane peeling. Am J Opbthalmol. 2002;133:89-94.

21. Yam HF, Kwok AK, Chan KP, et al. Effect of indocyanine green and illumination on gene expression in human pigment epithelial cells. Invest Opbthalmol Vis Sci. 2003;44:370-377.

22. Enaida H, Sakamoto T, Hisatomi T, et al. Morphological and functional damage of the retina caused by intravitreous indocyanine 
green in rat eyes. Graefes Arch Clin Exp Ophthalmol. 2002;240 209-213.

23. Sheidow TG, Blinder KJ, Holekamp N, et al. Outcome results in macular hole surgery: an evaluation of internal limiting membrane peeling with and without indocyanine green. Ophthalmology. 2003;110:1697-1701.

24. Mavrofrides E, Smiddy WE, Kitchens JW, et al. Indocyanine greenassisted internal limiting membrane peeling for macular holes: toxicity? Retina. 2006;26:637-644.

25. Kumagai K, Furukawa M, Ogino N, et al. Long-term outcomes of internal limiting membrane peeling with and without indocyanine green in macular hole surgery. Retina. 2006;26:613-617.

26. Schaal S, Barr CC. Management of macular holes: a comparison of 1-year outcomes of 3 surgical techniques. Retina. 2009;29:1091-1096.

27. Mitamura Y, Ohtsuka K. Relationship of dissociated optic nerve fiber layer appearance to internal limiting membrane peeling. Ophthalmology. 2005;112:1766-1770.

28. Narayanan R, Kenney MC, Kamjoo S, et al. Trypan blue: effect on retinal pigment epithelial and neurosensory retinal cells. Invest Ophthalmol Vis Sci. 2005;46:304-309.

29. Kodjikian L, Richter T, Halberstadt M, et al. Toxic effects of indocyanine green, infracyanine green, and trypan blue on the human retinal pigmented epithelium. Graefes Arch Clin Exp Ophthalmol. 2005;243:917-925.

30. Rezai KA, Farrokh-Siar L, Gasyna EM, et al. Trypan blue induces apoptosis in human retinal pigment epithelial cells. Am J Ophthalmol. 2004;138:492- 495 .

31. Foos RY. Vitreoretinal juncture; topographical variations. Invest Ophthalmol. 1972;11:801-808.

32. Brazitikos PD, Katsimpris JM, Tsironi E, Androudi S. Retinal nerve fiber layer thickness evaluation after trypan blue-assisted macular surgery. Retina. 2010;30:640-647.

33. Imai $\mathrm{H}$, Ohta $\mathrm{K}$. Microperimetric determination of retinal sensitivity in areas of dissociated optic nerve fiber layer following internal limiting membrane peeling. Jpn J Ophthalmol. 2010;54:435-440.
34. Kim CY, Lee JH, Lee SJ, et al. Visual field defect caused by nerve fiber layer damage associated with an internal limiting lamina defect after uneventful epiretinal membrane surgery. Am J Ophthalmol. 2002;133:569-571.

35. Terasaki H, Miyake Y, Nomura R, et al. Focal macular ERGs in eyes after removal of macular ILM during macular hole surgery. Invest Ophthalmol Vis Sci. 2001;42:229-234.

36. Tari SR, Vidne-Hay O, Greenstein VC, Barile GR, Hood DC, Chang S. Functional and structural measurements for the assessment of internal limiting membrane peeling in idiopathic macular pucker. Retina. 2003;27:567-572.

37. Smiddy WE, Feuer W, Cordahi G. Internal limiting membrane peeling in macular hole surgery. Ophthalmology. 2001;108:14711478.

38. Lois $\mathrm{N}$, Burr $\mathrm{J}$, Norrie $\mathrm{J}$, et al. Internal limiting membrane peeling versus no peeling for idiopathic full-thickness macular hole: a pragmatic randomized controlled trial. Invest Ophthalmol Vis Sci. 2011;52:1586-1592.

39. Margherio AR. Macular hole surgery in 2000. Curr Opin Ophthalmol. 2000;11:186-190.

40. Tadayoni R, Gaudric A, Haouchine B, Massin P. Relationship between macular hole size and the potential benefit of internal limiting membrane peeling. Br J Ophthalmol. 2006;90:12391241.

41. Ip MS, Baker BJ, Duker JS, et al. Anatomical outcomes of surgery for idiopathic macular hole as determined by optical coherence tomography. Arch Ophthalmol. 2002;120:29-35.

42. Freeman WR, Azen SP, Kim JW, et al. Vitrectomy for the treatment of full thickness stage 3 or 4 macular holes: results from a prospective randomized clinical trial. Arch Ophthalmol. 1997;115: $11-21$.

43. Bensen WE, Cruickshanks KC, Fong DS, et al. Surgical management of macular holes: a report by the American Academy of Ophthalmology. Ophthalmology. 2001;108:1328-1335. 